\title{
Optical and Energetic Investigation of an Advanced Corona Ignition System in a Pressure-Based Calorimeter
}

\author{
Valentino Cruccolini ${ }^{1,}{ }^{*}$, Gabriele Discepoli ${ }^{1}$, Federico Ricci $^{1}$, Carlo Nazareno Grimaldi ${ }^{1}$ \\ and Alessio Di Giuseppe ${ }^{2}$ \\ ${ }^{1}$ Università degli Studi di Perugia, Department of Engineering, 06125 Perugia (PG), Italy \\ ${ }^{2}$ Federal-Mogul Powertrain Italy a Tenneco Group Company, Stabilimento Ignition, 41012 Carpi \\ (MO), Italy
}

\begin{abstract}
In recent years, radio-frequency corona igniters have been extensively studied for their capability to ensure an effective ignition also in lean or diluted mixtures. Corona ignition is volumetric, with streamers coming from a star-shaped electrode. During the discharge, many radicals and excited species, able to speed up the combustion onset, are generated. At the same time, corona igniters are able to release a considerable amount of thermal energy inside the combustion chamber. The correct determination of such energy is crucial to evaluate the effectiveness of the ignition. In this work, corona discharge is experimentally evaluated inside an optical vessel. In this apparatus, the released thermal energy is measured by means of pressure-based calorimetry, and at the same time the natural luminosity of the streamers is recorded with a high-speed camera. The goal is to find a relationship between thermal energy release and streamers luminosity. Tests are performed using nitrogen as medium, at different pressure levels inside the vessel. The peak electrode voltage is varied to characterize the igniter behaviour in different operating conditions. The results of this work can be used to quantify the corona ignition capabilities to involve a wide amount of medium while releasing a high amount of thermal energy. A repeatability evaluation of streamer evolution is investigated as well.
\end{abstract}

\section{Introduction}

Internal combustion engines worldwide regulations have become more and more stringent, forcing to decrease the amount of pollutant emissions and, at the same time, to increase the thermal efficiency [1]. In literature many studies report the efforts to deal with these two aspects [2-4]. As for spark ignition (SI) engines, engine research community and manufacturers agree in considering a $45 \%$ brake thermal efficiency a feasible goal [5], ascertained that boosting, lean combustion and high EGR dilution are mandatory. In these conditions, the main barrier is represented by ignition, since with conventional spark plugs

* Corresponding author: valentino.cruccolini@unipg.it 
a robust flame kernel generation is hard to achieve. To improve ignition capability, sparkbased solutions like high-energy discharge or multiple strikes discharge [6] have shown their limits, since they have to deal with the reduced sparkplug lifetime [7]. Solutions based on the addition of highly-reactive species in the air-fuel mixture, like hydrogen [8], would have an excessive impact on fuel infrastructure [9]. Instead, interesting technologies to improve the stability of combustion initiation for boosted engines in lean and diluted environments are the pre-chamber ignition [10] and the low-temperature plasma ignition [11]. Regarding the latter, low-temperature or non-equilibrium plasma is able to enhance the combustion not only via thermal effect (the predominant in the spark discharge), but also via kinetic and transport effects [11]. Among several low-temperature plasma technologies, such as nanosecond-pulsed discharge [12], microwave discharge [13] and dielectric-barrier discharge [14], radio-frequency (RF) corona discharge is one of the most promising [15]. Several works showed its capability to improve lean or EGR-dilution tolerance $[8,16]$. The quick generation of a considerable amount of excited species, e.g. atomic oxygen, during the corona discharge is fundamental to explain the high capability of this system to speed up the combustion onset [17]. Calorimetry tests showed also that the thermal energy released during the corona discharge can be higher than the one released by a conventional spark igniter $[18,19]$. The corona discharge is based on the generation of streamers from a star-shaped electrode fed with RF power at the resonance frequency of the igniter [20]. There are evidences that link the released thermal energy, the intensity of streamers and the generation of radicals and excited species [21] for non-equilibrium plasma igniters, depending on the discharge parameters and on the in-cylinder medium. So far, anyway, studies on RF corona igniters have only been aimed at determining the combustion effectiveness [22], or the repeatability and the randomness of the streamer discharge [23], or the amount of the sole thermal energy [18].

This work, instead, reports the efforts to link an energetic information, i.e. the released thermal energy obtained via pressure-based calorimetry, and an optical information, i.e. the natural luminosity of the streamers during the RF corona discharge. The results of this work can be of great interest, since the relationship between thermal energy and streamer light could be potentially derived and used in optical engines, where only the discharge luminosity can be measured, the pressure-based calorimetry being not feasible.

\section{Experimental Setup and Methods}

\subsection{RF corona igniter}

In this work, a Tenneco ACIS (Advanced Corona Ignition System) streamer-type igniter is used (Fig. 1 left). It is featured with a star-shaped electrode, composed of 4 nominally identical tips, while the engine combustion chamber, in particular the piston, acts as the grounded counter-electrode. The igniter is supplied by a dedicated control unit with RF power at about $1.04 \mathrm{MHz}$, corresponding to the resonance frequency of the equivalent RLC circuit [20]. The discharge can be managed by calibrating the control unit, as explained in details in [18,22]: the driving voltage $V_{d}$ controls the peak electrode voltage $V_{e}$ (the relationship between them is linear [18]), while the discharge duration $\mathrm{T}_{\mathrm{d}}$ directly controls the time the corona discharge is active. Given the medium pressure, there is a precise $\mathrm{V}_{\mathrm{e}}$ range for which the corona discharge occurs. The lower limit is the corona inception voltage (CIV, according to the nomenclature used in [24]): below the CIV, the discharge does not occur. The upper limit is the breakdown voltage (BDV [24]), which implies a transition from non-equilibrium plasma streamers to thermal near-equilibrium plasma, typical of spark igniters. 
This igniter was already tested by our research group in an optical engine $[8,15,22,23,25]$ and in another calorimeter $[18,19]$, different from the one used in this work since there was no optical access.
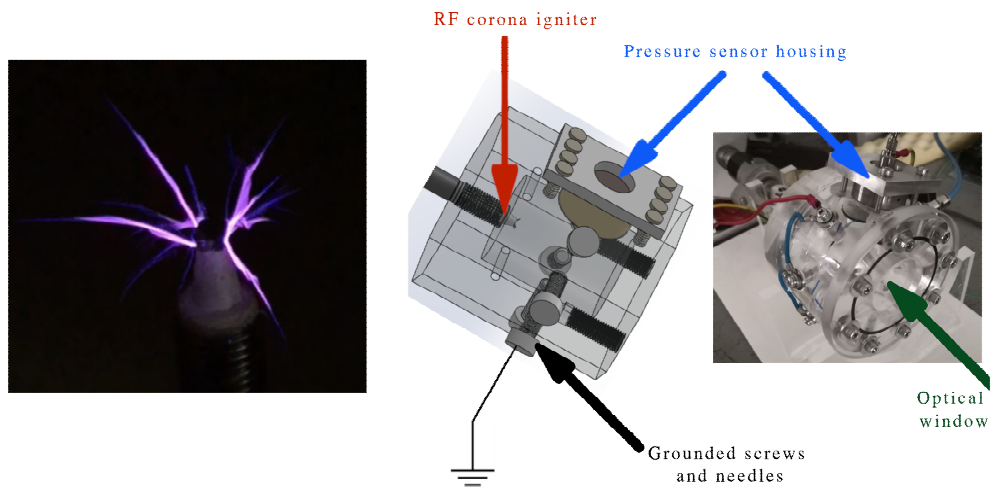

Fig. 1. Left: RF corona streamers generated in air at atmospheric pressure by a Tenneco ACIS streamer-type igniter. Right: CAD of the vessel and real vessel. Igniter, optical access, housing of the pressure sensor and grounded screws are pointed out.

\subsection{Optical calorimeter}

A constant-volume optical vessel in Plexiglas (to ensure low thermal conductivity, $0.187 \mathrm{~W} \cdot \mathrm{m}^{-1} \cdot \mathrm{K}^{-1}$ ) has been designed and realized to perform this experimental campaign. The inner volume $V_{c h}$ is $65.2 \mathrm{~cm}^{3}$ and the shape is cylindrical. The igniter is installed opposite to the optical window, while a piezoelectric sensor is placed in the lateral surface, together with grounded needles to direct the streamers (Fig. 1 right).

The vessel can be filled with gas up to 10 bar absolute of pressure. In this work, pure nitrogen from a cylinder (5.0 purity, Linde) at room temperature $(293 \pm 1 \mathrm{~K})$ is used. Details on the pressure control system can be found in the previous calorimetry works $[18,19]$. The discharge inside the vessel is triggered by a TTL signal, $10 \mathrm{~Hz}$ frequency, produced by an arbitrary wave Generator $H P$ 33120A. The current supplied to the igniter coil is measured by a Teledyne LeCroy CPO30 current probe (max continuous current input $30 \mathrm{~A}$, accuracy $\pm 1.5 \%$ full scale). The corona discharge event generates a pressure rise which is detected by means of a Kistler 7261 piezoelectric sensor (sensitivity $2200 \mathrm{pC} \cdot$ bar $^{-1}$, resolution of about $10^{-5}$ bar), whose signal is amplified and converted into a proportional voltage signal by a Kistler $5011 B$ charge amplifier. All the aforementioned signals are acquired by a fast oscilloscope Teledyne LeCroy Wavesurfer 3000 (Fig. 2, left) with a sampling frequency of $5 \mathrm{MHz}$, about five times bigger than ACIS supply frequency. For each test point, 100 consecutive events are recorded and stored.

A Phantom V710 high-speed camera with a Nikkor 50-mm f/1.4 lens (Fig. 2, left) is placed in front of the calorimeter to record the streamer luminosity. A 4-diopters close-up lens is placed downstream of the 50 - $\mathrm{mm}$ lens to improve the spatial resolution by reducing the minimum focal distance. In Table 1 the main features of the high-speed camera are reported. The camera records greyscale frames (Fig. 2, right), 8-bit depth. With the selected framerate and the discharge duration of $300 \mu \mathrm{s}$, the streamers are expected to be captured in a number of frames between 23 and 24 for each event. 

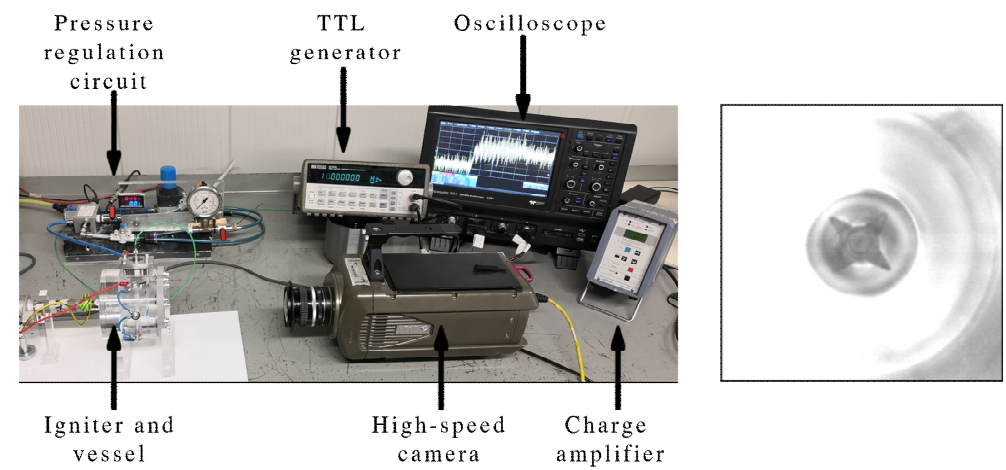

Fig. 2. Left: experimental layout. Right: high-speed camera view inside the vessel. Each side corresponds to about $25.6 \mathrm{~mm}$. The ACIS 4-tips central electrode is clearly visible.

Table 1. High-speed camera settings.

\begin{tabular}{|c|c|c|}
\hline Feature & Unit & Value \\
\hline Image resolution & pixel & $256 \times 256$ \\
\hline Sampling rate & $\mathrm{kfps}$ & 79 \\
\hline Exposure time & $\mu \mathrm{s}$ & 12.16 \\
\hline Bit depth & bit & 8 \\
\hline Spatial resolution & $\mu \mathrm{m}^{2} /$ pixel & 100 \\
\hline Temporal resolution @ $\mathrm{T}_{\mathrm{d}}=300 \mu \mathrm{s}$ & $\mathrm{frames} / \mathrm{event}$ & 23.7 \\
\hline Number of consecutive events recorded & - & 63 \\
\hline
\end{tabular}

The camera sensor is featured with a good sensitivity in the visible range of the electromagnetic spectrum, which is found to be adequate for the streamer detection: the corona discharge luminosity is related to the first negative system (FNS) of $\mathrm{N}_{2}^{+}\left(\mathrm{B}^{2} \Sigma_{\mathrm{u}}^{+}\right)$at about $391 \mathrm{~nm}$ [26], mainly in the streamer head, which moreover justifies the violet colour of the streamers (Fig. 1 left).

\subsection{Released energy calculation}

An in-house post-processing algorithm in Matlab allows to determine the thermal energy released by the corona discharge events. The pressure signal is filtered with a $2 \mathrm{kHz}$ low-pass filter to reduce the noise contribution [27]. The pressure difference $\Delta \mathrm{p}_{\text {ch }}$ due to the $300 \mu$ s discharge is directly proportional to the released thermal energy $\mathrm{Er}$, in agreement with the first law of thermodynamics by assuming the vessel adiabaticity [28]:

$$
\mathrm{E}_{\mathrm{r}}=(\gamma-1)^{-1} \cdot \mathrm{V}_{\mathrm{ch}} \cdot \Delta \mathrm{p} \mathrm{ch}
$$

where $\gamma$ is the specific heat ratio and $V_{c h}$ is the calorimeter chamber volume. This relationship can be considered as valid only for few milliseconds after the end of the discharge [18]. Details on the workflow to obtain $\mathrm{E}_{\mathrm{r}}$ from raw pressure signals are reported in $[18,19]$. Er is analysed for all the 100 consecutive strikes recorded at each operating point tested. 


\subsection{Streamer luminosity calculation}

For each test point, 63 consecutive corona events are recorded by the high-speed camera in a movie file whose format is the proprietary ".cine". A common trigger allows to match pressure rise and streamer evolution. Each "cine" file is composed of 35 frames: the first 10 are stored to characterize the background level, since no discharge event occurs on them; the following 24 refer to the discharge, while the last frame is used to confirm the corona end.

A postprocessing procedure developed via in-house Matlab scripts allows to compute the average grey level LgAvg of the frame. Most of the steps, described in Fig. 3a, are in common with a previous optical work aimed at characterizing streamer repeatability in an optical engine [23]. The procedure is the following:

- Background noise characterization: the average grey level value of each pixel in the 10 initial frames with no discharge is computed. This allows to obtain a map of the average noise in the 256x256 pixel matrix before the discharge.

- Background removal: for each discharge frame, noise is removed by subtracting, pixel-by-pixel, the average background map.

- Equalization: grey levels are no longer displayed in the 0-255 range (8-bit resolution), but only in the $0-100$ range. This choice allows to highlight the branching structure of the streamers, improving the comprehensibility, and this is found to be effective for all the different cases.

- Filtering: a 2D Gaussian filter with a standard deviation $\sigma=1$ is applied to reduce the residual noise. The $\sigma$ value is a good compromise between noise cancellation and boundary conservation.

- Thresholding: a fixed threshold is applied to binarize the frame, i.e. to convert the greyscale image into a black-\&-white one. The threshold application is also a way to determine if an image contains pixels that are bright enough to consider as active the corona discharge: a frame with 0 pixels above the threshold means that there are no streamers.

- Average grey level: only for the frames in which the discharge is present (at least one pixel over the threshold), the average grey level of the entire frame ( $\mathrm{LgAvg}$, Frame) is computed. This value is a measure of the luminous emission of the streamer in a period corresponding to the sensor exposure time, so it represents the average brightness of the frame.

This procedure is repeated for all the consecutive frames of the same event. The average grey level of a single event (LgAvg,Event) is computed by averaging LgAvg,Frame from frame \# 10 (the last before the start of the event) to frame \# 35 (the first for which the corona is certainly over).

The corona discharge being a stochastic event, a certain variability among the consecutive events is expected: in Fig. $3 b$ the LgAvg,Frame trend over the discharge for 63 events of the same series is reported. The behaviour of the consecutive events is globally similar, and in the same figure the average grey level evolution over frames is plotted in green solid line. From this dataset, the average grey level LgAvg,Series (red dashed line) can be computed, together with the corrected-series standard deviation LgStd,Series. Note that, ultimately, LgAvg,Series represents the average luminosity produced by an entire series of corona events at the same operating conditions (pressure, driving voltage): it can be then compared to the energy released by the igniter. 


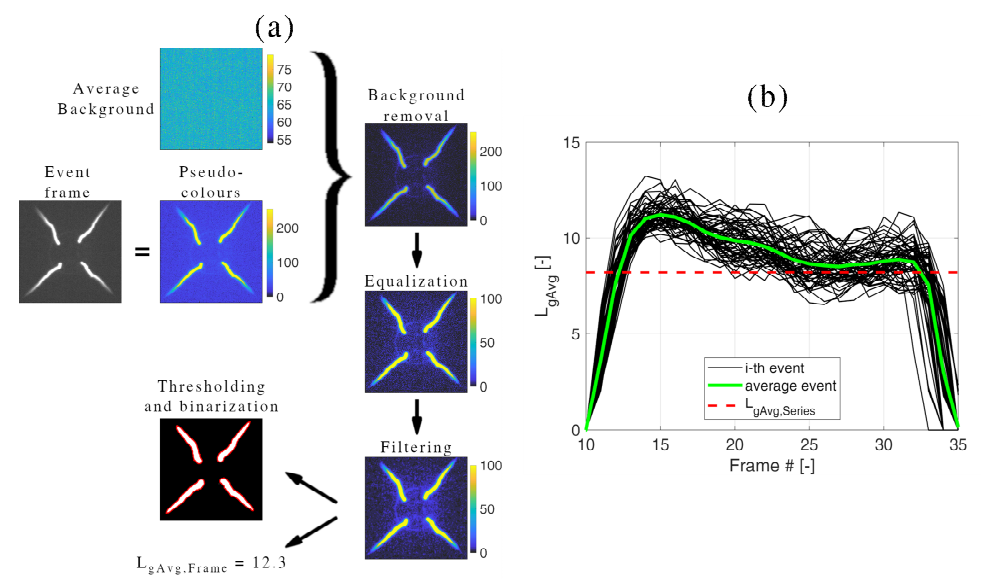

Fig. 3. (a) Image postprocessing workflow to obtain the frame average grey level LgAvg,Frame. (b) LgAvg,Frame evolution for all the 63 consecutive events of a test point (black lines), series average frameby-frame (green solid line), and the corresponding whole series average level Lg,Avg,Series (red dashed line).

\subsection{Test campaign}

Tests are performed in $\mathrm{N}_{2}$ at three different pressure levels ( $\mathrm{p}_{\mathrm{ch}}=1,2$ and 3 bar absolute) and two different driving voltages $\left(\mathrm{V}_{\mathrm{d}}=16\right.$ and $\left.20 \mathrm{~V}\right)$. The latter values correspond to a peak electrode voltage $\mathrm{V}_{\mathrm{e}}$ of, respectively, about 8.2 and $10.7 \mathrm{kV}$, regardless of the pressure [18]. The corona duration $\mathrm{T}_{\mathrm{d}}$ is fixed at $300 \mu \mathrm{s}$, such as in previous calorimetry tests [18]. $300 \mu \mathrm{s}$ is also an effective value to normally ensure the ignition in internal combustion engines [22]. The experimental campaign is composed of 6 different test points, identified by a name in the form " $\mathrm{p}_{\mathrm{ch}} \mathrm{V}_{\mathrm{d}}$ " so they can be expressed as 1_16, 1_20, 2_16, 2_20, 3_16 and 3_20.

\section{Results}

In Table 2 the main results of the pressure-based calorimetry can be found. In the second and third columns, respectively, the mean thermal energy value $\left(\mathrm{E}_{\mathrm{ravg}}\right)$ and the corrected standard deviation (Erstd) are reported. As expected [18], an ErAvg rising trend with voltage at fixed pressure is found. The energy reduction with pressure, instead, is found to be strongly dependent on the voltage. In particular, at $\mathrm{V}_{\mathrm{d}}=20 \mathrm{~V}$, the energy values at 2 and 3 bar are very close each other, while from 1 to 2 bar the gap is higher. At $V_{d}=16 \mathrm{~V}$, instead, energy values are more "evenly-distributed" among the 3 pressure levels. This denotes a different behaviour of the $\operatorname{Er}\left(\mathrm{V}_{\mathrm{d}}\right)$ relationship with pressure, a phenomenon already found in [18]. Er results are close to the ones in [18] with similar pressure and similar $\mathrm{V}_{\mathrm{d}}$ and $\mathrm{T}_{\mathrm{on}}$, thus confirming the high reproducibility of the measurements. At engine relevant pressure levels, higher than the ones tested in this work, Er can reach up to $80 \mathrm{~mJ}$ $[18,19]$ depending on the discharge parameters. Finally, and just as a comparison, the expected thermal energy with a conventional spark plug with a pressure range of 1-3 bar would reach only about 1-2 mJ [19,28].

The optical results can be found as well in Table 2, fourth and fifth columns. The trend of the average values is similar to what found in the energy cases. The standard deviation, which turns out to be almost the same for the two techniques, confirms the strong correlation between the two phenomena. Ultimately, both thermal energy and discharge 
brightness rise with voltage and decrease with pressure, coherently with the nonequilibrium plasma physics in the corresponding range of reduced electric field, the discharge key parameter [29].

Table 2. Optical calorimeter results

\begin{tabular}{|c|c|c|c|c|}
\hline Series $\left(p_{c h} \_V_{d}\right)$ & ErAvg $[\mathbf{m J}]$ & $\mathbf{E r S t d}_{\mathbf{m}}[\mathbf{m J}]$ & LgAvg,Series [-] & LgStd,Series [-] \\
\hline 1_16 & 11.4 & 0.05 & 10.6 & 0.06 \\
\hline 1_20 & 19.4 & 0.11 & 19.4 & 0.10 \\
\hline 2_16 & 8.1 & 0.03 & 7.1 & 0.05 \\
\hline 2_20 & 16.2 & 0.04 & 12.2 & 0.06 \\
\hline 3_16 & 5.9 & 0.11 & 4.0 & 0.14 \\
\hline 320 & 15.9 & 0.06 & 8.2 & 0.05 \\
\hline
\end{tabular}

\section{Discussion}

\subsection{Thermal Energy and Luminosity}

(a)

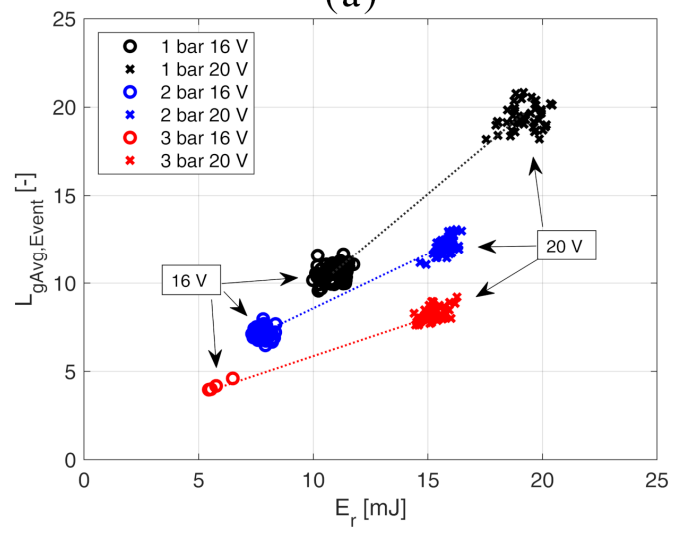

(b)

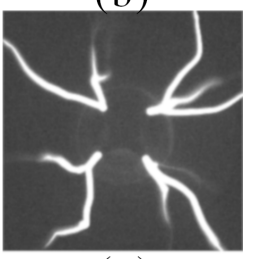

(c)

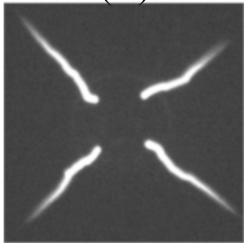

Fig. 4. (a) Relationship between the released thermal energy and the average grey level for each corona event. Series are represented in a different way according to pressure (black $=1$ bar, blue $=2$ bar, red $=3$ bar) and driving voltage (circles $=16 \mathrm{~V}$, crosses $=20 \mathrm{~V}$ ). Lines that link the series with the same pressure are reported in dashed lines only for sake of clarity. (b) 1_20 frame. (c) 3_20 frame.

In Fig. 4a the distribution of energy and average luminosity for the same corona event is reported, for all the series. The 3_16 trend is featured with only few values (only the ones with the highest energy), and a focus on them can be found in the next section.

At a given pressure value, higher amounts of both released energy and streamer luminosity are found moving towards high $\mathrm{V}_{\mathrm{d}}$. Anyway, similar values of released energy do not imply similar values of luminosity: there are cases with almost the same $\mathrm{E}_{\mathrm{r}}$ and a very different luminous emission (e.g. 2_20 and 3_20) and others with same LgAvg,Series (e.g. 1_16 and 2_20) but different energy. This because the lines that link the iso-pressure series, 
featured with positive slope, result to be pressure-dependent: the higher the pressure, the lower the slope.

This can be explained by considering that lower pressure levels result in an increase in streamer extent, thickness and branching (Fig. 4b), so that each frame is brighter than the corresponding high-pressure one, which is on average more narrow (Fig. 4c). Moreover, in several occurrences of the 1_20 series the streamers are found to go beyond the frame area (Fig. 4b): this implies that such frames are particularly brighter than the ones in which streamers are all confined into the frame area. This is not found in the other series (Fig. 4c). A possible countermeasure, i.e. slightly moving away the camera from the vessel, would affect the spatial resolution and for this reason we did not change the camera position, accepting to underestimate a limited number of occurrences.

Ultimately, the pressure level has an influence in the luminous emission, while it does not affect the way the pressure-based calorimetry detects $\mathrm{E}_{\mathrm{r}}$.

\subsection{Inception voltage case}

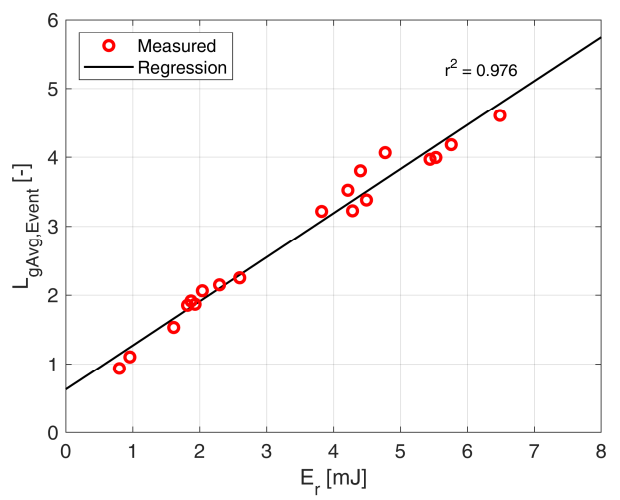

Fig. 5. Focus on CIV (3_16 series), where also the occurrences with a partial level of energy are considered. Red markers: experimental points, black solid line: regression line.

The 3_16 point is very close to the corona inception conditions. Since the inception process is characterized by high stochasticity, the events show off very different energy and brightness levels in close proximity to CIV. In many occurrences the discharge is not able to start at all, in other cases it starts later, in other ones not all the 4 electrode tips are interested in the generation of the streamers, as already found in [18]. The results for the 3 _16 case in Table 2 and in Fig. 4 refer only to the high-energy occurrences, but, if all the occurrences but the zero-energy ones are considered (Fig. 5), a strong linearity between LgAvg,Event and $\mathrm{E}_{\mathrm{r}}$ can be found (the regression line is featured with an $\mathrm{r}^{2}$ of about $98 \%$ ).

Finally, note that the experimental points of the CIV tend to distribute not continuously but in groups, suggesting the existence of discrete energetic levels, which justifies the binning procedure reported in [18].

\section{Conclusions}

This work reports an analysis based on both imaging and pressure-based calorimetry on RF corona discharges generated by a Tenneco streamer-type ACIS igniter. An optical vessel has been specifically realized for this work: pressure rise and streamer luminosity of 
each corona event can be simultaneously acquired. Tests are performed in pure nitrogen at three pressure levels (1, 2 and 3 bar absolute) and 2 peak voltage levels at the electrode.

The main findings are listed in the following:

- The thermal energy obtained from pressure-based calorimetry is in agreement with previous measurements on a different calorimeter.

- Both thermal energy and discharge brightness rise with an increase of voltage or a decrease of medium pressure. This is coherent with the physics of the nonequilibrium plasma in the corresponding range of reduced electric field.

- The statistical distribution of streamer luminosity and released energy over the same series of events is very similar, confirming that the two phenomena are linked.

- Given the pressure, the event luminosity rises linearly with the released thermal energy. This is demonstrated for the 3_16 point, close to the CIV, in which a large number of energy levels and brightness levels is found.

- The linear relationship between event luminosity and thermal energy depends also on chamber pressure. A given rise in thermal energy results in a corresponding rise in luminosity which is higher at 1 bar than at 3 bar.

\section{Glossary and nomenclature}

$\begin{array}{ll}\text { ACIS } & \text { advanced corona ignition system } \\ \text { Avg } & \text { average } \\ \text { BDV } & \text { breakdown voltage } \\ \text { CIV } & \text { corona inception voltage } \\ \mathrm{Er}_{\mathrm{r}} & \text { released thermal energy } \\ \mathrm{LgAvg} & \text { average grey level (of a frame, of an event, of a series) } \\ \mathrm{Std} & \text { corrected-series standard deviation } \\ \mathrm{T}_{\mathrm{d}} & \text { corona discharge duration } \\ \mathrm{V}_{\mathrm{d}} & \text { corona driving voltage } \\ \mathrm{V}_{\mathrm{e}} & \text { peak electrode voltage } \\ \Delta \mathrm{p}_{\mathrm{ch}} & \text { difference in chamber pressure before and after the corona discharge }\end{array}$

\section{Funding}

This research activity was partially granted by the Basic Research Funds supplied by the Department of Engineering of the University of Perugia

\section{References}

[1] T. Johnson and A. Joshi. "Review of Vehicle Engine Efficiency and Emissions", SAE Int. J. Engines 11, 2018-01-0329 (2018)

[2] D. Takahashi et al. "Combustion Development to Realize High Thermal Efficiency Engines", SAE Int. J. Engines 9, 2016-01-0693 (2016)

[3] J. Zembi et al. "CFD Analysis of Port Water Injection in a GDI Engine under Incipient Knock Conditions", Energies 12, 3409 (2019)

[4] M. Battistoni et al. "Assessment of Port Water Injection Strategies to Control Knock in a GDI Engine through Multi-Cycle CFD Simulations", in SAE Tech. Pap. (2017). doi:10.4271/2017-24-0034

[5] K. Nakata et al. "Engine Technologies for Achieving 45\% Thermal Efficiency of S.I. Engine", SAE Int. J. Engines 9, 2015-01-1896 (2015)

[6] D. Jung and N. Iida. "An investigation of multiple spark discharge using multi-coil ignition 
system for improving thermal efficiency of lean SI engine operation", Appl. Energy 212, 322-332 (2018)

[7] D. Breden et al. "High-Fidelity Numerical Modeling of Spark Plug Erosion", SAE Tech. Pap. Ser. 1, 1-12 (2019)

[8] V. Cruccolini et al. "Lean combustion analysis using a corona discharge igniter in an optical engine fueled with methane and a hydrogen-methane blend", Fuel 259, 116290 (2020)

[9] G. Kalghatgi. "Is it really the end of internal combustion engines and petroleum in transport?", Appl. Energy 225, 965-974 (2018)

[10] J. Benajes et al. "Evaluation of the passive pre-chamber ignition concept for future high compression ratio turbocharged spark-ignition engines", Appl. Energy 248, 576-588 (2019)

[11] Y. Ju and W. Sun. "Plasma assisted combustion: Dynamics and chemistry", Prog. Energy Combust. Sci. 48, 21-83 (2015)

[12] R. Scarcelli et al. "Modeling non-equilibrium discharge and validating transient plasma characteristics at above-atmospheric pressure", Plasma Sources Sci. Technol. 27, 124006 (2018)

[13] Y. Ikeda et al. "Development of Innovative Microwave Plasma Ignition System with Compact Microwave Discharge Igniter", in SAE Tech. Pap. (2015). doi:10.4271/2015-242434

[14] C. A. Idicheria et al. "An Advanced Ignition System for High Efficiency Engines", in Ignition Syst. Gasol. Engines 4th Int. Conf. December 6 - 7, 2018, Berlin, Ger. 40-54 (2018). doi:10.5445/IR/1000088317

[15] V. Cruccolini et al. "Comparative Analysis between a Barrier Discharge Igniter and a Streamer-Type Radio-Frequency Corona Igniter in an Optically Accessible Engine in Lean Operating Conditions", in SAE Tech. Pap. (2020). doi:10.4271/2020-01-0276

[16] D. I. Pineda et al. "Application of Corona Discharge Ignition in a Boosted Direct-Injection Single Cylinder Gasoline Engine: Effects on Combustion Phasing, Fuel Consumption, and Emissions", SAE Int. J. Engines 9, 2016-01-9045 (2016)

[17] R. Scarcelli et al. "Numerical simulation of a nano-pulsed high-voltage discharge and impact on low-temperature plasma igni-tion processes for automotive applications", in Ignition Syst. Gasol. Engines 4th Int. Conf. (2018). doi:10.5445/IR/1000088603

[18] G. Discepoli et al. "Experimental characterisation of the thermal energy released by a RadioFrequency Corona Igniter in nitrogen and air", Appl. Energy 263, 114617 (2020)

[19] G. Discepoli et al. "Experimental assessment of spark and corona igniters energy release", Energy Procedia 148, 1262-1269 (2018)

[20] J. Burrows and K. Mixell. "Analytical and Experimental Optimization of the Advanced Corona Ignition System", in Ignition Syst. Gasol. Engines 267-292 (Springer International Publishing, 2016). doi:10.1007/978-3-319-45504-4_17

[21] B. M. Wolk and I. Ekoto. "Calorimetry and Imaging of Plasma Produced by a Pulsed Nanosecond Discharge Igniter in EGR Gases at Engine-Relevant Densities", SAE Int. J. Engines 10, 2017-01-0674 (2017)

[22] A. Cimarello et al. "Combustion Behavior of an RF Corona Ignition System with Different Control Strategies", in SAE Tech. Pap. vols 2018-April 1-19 (2018).

[23] V. Cruccolini et al. "An Optical Method to Characterize Streamer Variability and Streamerto-Flame Transition for Radio-Frequency Corona Discharges", Appl. Sci. 10, 2275 (2020)

[24] H. Toyota et al. "Gaseous electrical discharge characteristics in air and nitrogen at cryogenic temperature", IEEE Trans. Dielectr. Electr. Insul. 9, 891-898 (2002)

[25] A. Cimarello et al. "Analysis of RF Corona Ignition in Lean Operating Conditions Using an Optical Access Engine", in SAE Tech. Pap. (2017). doi:10.4271/2017-01-0673

[26] T. Hoder et al. "Sub-nanosecond delays of light emitted by streamer in atmospheric pressure

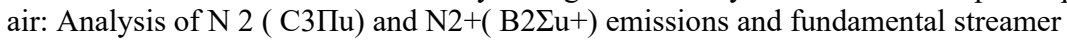
structure", J. Appl. Phys. 117, 073302 (2015)

[27] F. Ricci et al. "Experimental and Numerical Investigations of the Early Flame Development Produced by a Corona Igniter", in SAE Tech. Pap. Ser. vol. 1 (2019).

[28] Z. Abidin and C. Chadwell. "Parametric Study and Secondary Circuit Model Calibration Using Spark Calorimeter Testing", in SAE Tech. Pap. (2015). doi:10.4271/2015-01-0778

[29] N. Y. Babaeva and G. V. Naidis. "On streamer dynamics in dense media", J. Electrostat. 53, 123-133 (2001) 